\title{
Postpartum plasma metabolomic profile among women with preeclampsia and preterm delivery: implications for long-term health
}

Xiumei Hong ${ }^{1 *+} \mathbb{D}$, Boyang Zhang ${ }^{2 \dagger}$, Liming Liang ${ }^{3,4}$, Yan Zhang ${ }^{2}$, Yuelong $\mathrm{Ji}^{1}$, Guoying Wang ${ }^{1}$, Hongkai $\mathrm{Ji}^{2}$, Clary B. Clish ${ }^{5}$, Irina Burd ${ }^{6}$, Colleen Pearson 7 , Barry Zuckerman ${ }^{7}$, Frank B. $\mathrm{Hu}^{3,8,9}$ and Xiaobin Wang ${ }^{1,10}$

\begin{abstract}
Background: Preeclampsia and preterm delivery (PTD) are believed to affect women's long-term health including cardiovascular disease (CVD), but the biological underpinnings are largely unknown. We aimed to test whether maternal postpartum metabolomic profiles, especially CVD-related metabolites, varied according to PTD subtypes with and without preeclampsia, in a US urban, low-income multi-ethnic population.

Methods: This study, from the Boston Birth Cohort, included 980 women with term delivery, 79 with medically indicated PTD (mPTD) and preeclampsia, 52 with mPTD only, and 219 with spontaneous PTD (SPTD). Metabolomic profiling in postpartum plasma was conducted by liquid chromatography-mass spectrometry. Linear regression models were used to assess the associations of each metabolite with mPTD with preeclampsia, mPTD only, and SPTD, respectively, adjusting for pertinent covariates. Weighted gene coexpression network analysis was applied to investigate interconnected metabolites associated with the PTD/preeclampsia subgroups. Bonferroni correction was applied to account for multiple testing.

Results: A total of 380 known metabolites were analyzed. Compared to term controls, women with mPTD and preeclampsia showed a significant increase in 36 metabolites, mainly representing acylcarnitines and multiple classes of lipids (diacylglycerols, triacylglycerols, phosphocholines, and lysophosphocholines), as well as a decrease in 11 metabolites including nucleotides, steroids, and cholesteryl esters (CEs) $\left(P<1.3 \times 10^{-4}\right)$. Alterations of diacylglycerols, triacylglycerols, and CEs in women with $\mathrm{mPTD}$ and preeclampsia remained significant when compared to women with mPTD only. In contrast, the metabolite differences between women with mPTD only and term controls were only seen in phosphatidylethanolamine class. Women with sPTD had significantly different levels of 16 metabolites mainly in amino acid, nucleotide, and steroid classes compared to term controls, of which, anthranilic acid, bilirubin, and steroids also had shared associations in women with mPTD and preeclampsia.

(Continued on next page)
\end{abstract}

\footnotetext{
* Correspondence: xhong3@jhu.edu

${ }^{+}$Xiumei Hong and Boyang Zhang contributed equally to this work.

'Center on the Early Life Origins of Disease, Department of Population,

Family and Reproductive Health, Johns Hopkins University Bloomberg School

of Public Health, 615 N. Wolfe St, Baltimore, MD 21205-2179, USA

Full list of author information is available at the end of the article
}

(c) The Author(s). 2020 Open Access This article is licensed under a Creative Commons Attribution 4.0 International License, which permits use, sharing, adaptation, distribution and reproduction in any medium or format, as long as you give appropriate credit to the original author(s) and the source, provide a link to the Creative Commons licence, and indicate if changes were made. The images or other third party material in this article are included in the article's Creative Commons licence, unless indicated otherwise in a credit line to the material. If material is not included in the article's Creative Commons licence and your intended use is not permitted by statutory regulation or exceeds the permitted use, you will need to obtain permission directly from the copyright holder. To view a copy of this licence, visit http://creativecommons.org/licenses/by/4.0/ The Creative Commons Public Domain Dedication waiver (http://creativecommons.org/publicdomain/zero/1.0/) applies to the data made available in this article, unless otherwise stated in a credit line to the data. 


\begin{abstract}
(Continued from previous page)
Conclusion: In this sample of US high-risk women, PTD/preeclampsia subgroups each showed some unique and shared associations with maternal postpartum plasma metabolites, including those known to be predictors of future CVD. These findings, if validated, may provide new insight into metabolomic alterations underlying clinically observed PTD/preeclampsia subgroups and implications for women's future cardiometabolic health.
\end{abstract}

Keywords: Preterm delivery, Preeclampsia, Medically indicated preterm delivery, Spontaneous preterm delivery, Postpartum, Metabolome

\section{Background}

Pregnancy is believed to be a vulnerable time period for women's long-term health. While ample evidence suggests that preterm delivery (PTD) and other pregnancy complications such as preeclampsia are important risk factors for the health of the offspring, much less appreciated is that these same traits may also affect the longterm health of the mother. Pregnancy complications and PTD are associated with risk of having the same conditions in subsequent pregnancies, but also lead to an increased long-term risk of adverse health outcomes, including cardiovascular diseases (CVDs) [1-4], the leading causes of death for women in the USA [5].

All-cause PTDs are associated with a 1.5- to 3-fold higher risk of CVD $[1-4,6]$. Previous studies have shown that much of this association is accounted for by metabolic disorders during pregnancy, especially preeclampsia (which explains about $24-26 \%$ of the association) [1], or by metabolic disorders postpartum (which may explain about $13-15 \%$ of the association) [3]; however, a substantial portion of the risk remains unaccounted for. The PTD-CVD associations persist even in pregnancies uncomplicated by preeclampsia or hypertensive disorders $[3,6,7]$, indicating the need to explore other pathways via which PTD and CVD are linked. PTD is heterogeneous, with a variety of different PTD subtypes (i.e., spontaneous vs. medically indicated PTD), with and without preeclampsia, which may affect CVD risk through different pathways. However, such data are quite limited.

We hypothesized that women who experienced different PTD subtypes with and without preeclampsia may exhibit CVD risk factors or biomarkers early in the postpartum period, long before the development of CVD. The identification of such early biomarkers may provide new insight into the biological underpinning of the link between PTD/preeclampsia and women's future cardiometabolic health and provide targets for prevention and intervention in high-risk women. Metabolomic profiling, via the high-throughput assessment of a large number of circulating small molecule metabolites across multiple pathways, may offer advantages in elucidating biological pathways and biomarker discovery. Recent metabolomic studies have successfully identified multiple metabolites of different classes as early biomarkers for CVD, including branched-chain and aromatic amino acids (i.e., leucine, isoleucine, valine, phenylalanine, and tyrosine) [810], short- and medium-chain acylcarnitines [11-13], and metabolites of lipid classes [14-17]. Little is known about whether such metabolites vary in women with PTD vs. term delivery with or without preeclampsia.

In this study, by applying state-of-the-art metabolomic approaches, we quantified maternal metabolomic profiles in postpartum plasma (collected within 1-3 days after delivery) of 1411 women enrolled from the Boston Birth Cohort (BBC), an inner-city multi-ethnic longitudinal study cohort. We aimed to identify both shared and divergent metabolomic patterns (especially in those metabolites associated with CVD risk) for different PTD subtypes with and without preeclampsia, and further to test whether such PTD/preeclampsia-associated metabolomic patterns may vary by maternal characteristics such as ethnicity and parity.

\section{Methods \\ Study population}

The study population was a subset of the BBC, an ongoing longitudinal cohort study begun in 1998 at the Boston Medical Center in Massachusetts. Detailed information about the $\mathrm{BBC}$ has been reported elsewhere [18, 19]. Briefly, mothers who delivered singleton live births were eligible for the study and were invited to participate within 1 to 3 days after delivery. We excluded pregnancies that were a result of in vitro fertilization, multiple gestations, those with fetal chromosomal abnormalities or major birth defects, and preterm deliveries due to non-obstetric factors (such as trauma). The BBC is a low-income patient population, with a relatively high proportion of PTD and preeclampsia. After obtaining written informed consent, each mother was interviewed using a standardized questionnaire to gather dietary and epidemiologic data, and their electronic medical records (EMRs) were abstracted. Maternal blood samples were obtained within $24-72 \mathrm{~h}$ after delivery. The study protocol was approved by the Institutional Review Boards of Boston University Medical Center and the Johns Hopkins Bloomberg School of Public Health. 


\section{Definition of preterm delivery and preeclampsia}

Gestational age was assessed by early prenatal ultrasound ( $<20$ weeks) or based on the first day of the last menstrual period as recorded in maternal EMRs if early prenatal ultrasound was not available [19]. Medically indicated PTD (mPTD) was defined as a delivery by medical induction or Cesarean section (c-section) at $<37$ weeks without uterine contractions or rupture of membranes. The indications for medical induction or csection included gestational complication (mainly preeclampsia), placenta abruption, placenta previa, oligohydramnios, previous c-section, intrauterine growth restriction, absent end diastolic flow, not reassuring fetal heartbeat, fetal distress, signs of reduced amniotic fluid index, or persistent fetal tachycardia. Spontaneous PTD (sPTD) was defined as a delivery occurring secondary to documented active preterm labor (uterine contractions with cervical effacement and dilation at $<37$ weeks) or premature rupture of membranes at $<37$ weeks without uterine contractions or both. We further divided SPTD as early sPTD (spontaneous delivery at $<33$ weeks) or late SPTD (spontaneous delivery at $33-36^{6 / 7}$ weeks). Physician-diagnosed preeclampsia, eclampsia, and hemolysis, elevated liver enzymes, and low platelet syndromes (HELLP) were extracted from the maternal EMRs and were further confirmed by review of all relevant medical records to meet the definition of preeclampsia according to the recent American College of Obstetricians and Gynecologist (ACOG) criteria [20].

We classified all participating women into one of the following PTD/preeclampsia subgroups: (1) women of term delivery without preeclampsia (including physiciandiagnosed preeclampsia, eclampsia, and HELLP), as term controls; (2) women with both mPTD and preeclampsia; (3) women with mPTD but no preeclampsia (referred to as "mPTD only" hereafter); and (4) women with sPTD but no preeclampsia (referred to as "sPTD" hereafter, and further subdivided by early or late SPTD in supplemental analyses). Among the 1411 women with available metabolomic profiles, 9 were removed due to missing preeclampsia status and 72 (61 preeclamptic women with term delivery and 11 preeclamptic women with SPTD) were removed as they did not meet the criteria for either the control or the PTD subgroups as described above.

\section{Epidemiological and clinical data}

Using a standard maternal questionnaire interview [18], maternal epidemiological factors were collected including race/ethnicity, maternal age at delivery, highest education level, parity, smoking during pregnancy, alcohol drinking during pregnancy, illicit drug use during pregnancy, lifetime stress, previous history of PTD, and maternal birthplace (US-born/non-US-born). Maternal pregestational body mass index (BMI), calculated as selfreported weight $(\mathrm{kg})$ divided by height squared $\left(\mathrm{m}^{2}\right)$, was categorized into four groups: normal weight $(<25.0 \mathrm{~kg} /$ $\mathrm{m}^{2}$ ), overweight $\left(25-29.9 \mathrm{~kg} / \mathrm{m}^{2}\right)$, obesity $\left(\geq 30 \mathrm{~kg} / \mathrm{m}^{2}\right)$, and unknown. Clinical complication before pregnancy, including chronic hypertension and pregestational diabetes, was defined based on archived EMRs.

\section{Metabolomic profiles and data processing steps}

Maternal plasma samples, randomly distributed in each plate, were shipped to the Broad Institute of MIT and Harvard (Cambridge, MA, USA) for metabolomic profiling. Details of this technique can be found elsewhere [21, 22]. Briefly, two liquid chromatography tandem mass spectrometry techniques were applied, including hydrophilic interaction liquid chromatography (HILIC) analyses of water-soluble metabolites in the positive ionization mode and C8 chromatography with positive ion mode analyses of polar and non-polar plasma lipids. Metabolites in different lipid classes, including cholesterol esters (CEs), diacylglycerols (DAGs), triacylglycerols (TAGs), lysophosphatidylcholines (LPCs), lysophosphatidylethanolamines (LPEs), phosphatidylcholines (PCs), and phosphatidylethanolamines (PEs), were further classified based on the number of total acyl chain carbon atoms and double bond contents and were annotated as " $\mathrm{C}$ number of total acyl chain carbon atoms]:[number of double bonds in fatty acid meoeties] [lipid class]," accordingly.

A pooled plasma study reference sample, comprised from all of the studied plasma samples, was evenly distributed throughout the study samples (every $\sim 20$ study samples) as a quality control step. Using these reference samples, a coefficient of variance (CV) was calculated for each metabolite. Internal standard peak areas were monitored for quality control and to ensure system performance throughout the analyses. A total of 432 known metabolites were successfully quantified, and 40 metabolites with $\mathrm{CV}>20 \%, 10$ metabolites unquantifiable in $>10 \%$ participants, and 2 xenobiotics were removed from downstream analyses. The remaining 380 metabolites, with any non-detectable values being imputed as one half of the minimal value, were inverse normally transformed to render the distributions approximately Gaussian and to remove the impact of outliers.

\section{Data analyses}

Maternal characteristics were compared between each of the three PTD/preeclampsia subgroups (as defined above) and term controls, using ANOVA tests for continuous variables and $\chi^{2}$ tests (or Fisher's exact tests, when necessary) for categorical variables. To identify whether metabolites varied significantly in each PTD/ preeclampsia subgroup compared to term controls, we fit a linear regression model with each metabolite as the 
outcome and the four-categorical index variable (term controls/mPTD and preeclampsia/mPTD only/sPTD) as the exposure, adjusting for conventional and clinical covariates including maternal age at delivery, maternal ethnicity/race, maternal birthplace (US-born versus nonUS-born), pregestational BMI category, pregestational diabetes, chronic hypertension, marital status, highest education level, parity, maternal smoking during pregnancy, illicit drug use, lifetime stress, and fetal sex. Bonferroni correction was used to account for multiple testing.

We then performed weighted gene coexpression network analysis (WGCNA) using the WGCNA package in $\mathrm{R}$ [23], to reduce the number of comparisons made and minimize issues of metabolite collinearity. This method utilizes an unsupervised network-based approach to group metabolites into "modules" based on their correlation patterns. After hierarchical clustering, highly interconnected metabolites were assigned to the same module and was annotated as a unique color. The remaining metabolites $(n=51)$ that were not assigned to any of the established modules were removed from the subsequent analyses. For each module, the top hub metabolite was identified, which was the metabolite having the highest connectivity with other metabolites in the same module. Each participant was then assigned a "score" for each module, which was calculated as the first principal component of the metabolites within the module. The score for each module was then analyzed as the outcome in the subsequent analyses, using the linear regression models with the adjustment of covariates as described above. All analyses were performed using R Software, version 3.6.1.

\section{Results}

\section{Population characteristics of the study participants}

This study included 980 women with term delivery (or term controls) and 350 women with any of the following three PTD/preeclampsia subgroups: (1) $\mathrm{mPTD}$ and preeclampsia $(n=79),(2)$ mPTD only $(n=52)$, and (3) $\operatorname{sPTD}(n=219)$. The population characteristics across the four study groups are summarized in Table 1. Compared to term controls, women with both MPTD and preeclampsia were older and were more likely to be obese before pregnancy, be stressed, and have chronic hypertension and pregestational diabetes, while women with SPTD were more likely to be unmarried, to be USborn, to smoke during pregnancy, and to have illicit drug use than term controls (all $P<0.05$ ). As expected, c-section was significantly more common in women with $\mathrm{mPTD}$, either with $(65.8 \%)$ or without preeclampsia (80.8\%) than in term controls (31.8\%).

\section{Altered plasma metabolites in relation to different PTD/ preeclampsia subgroups}

Figure 1 and Table 2 present the metabolites that were significantly different from term controls in at least one PTD/preeclampsia subgroup. After Bonferroni correction for multiple testing $\left(P<1.3 \times 10^{-4}\right)$ and with term controls as the reference, women with $\mathrm{mPTD}$ and preeclampsia had significantly different patterns in 47 metabolites, including higher levels of 36 metabolites that were mainly amino acids $(n=7)$, total carnitine or acylcarnitines $(\mathrm{C} 2, \mathrm{C} 3, \mathrm{C} 5-\mathrm{DC}, \mathrm{C} 8, n=4)$, nucleotides $(n=$ 4), DAGs (C34:1, C34:1 DAG/TAG fragment, C34:2, C36:2, C36:3, C38:4, and C38:5, $n=7$ ), TAGs (C50:2, C52:2, C52:3, $n=3$ ), LPCs (C22:5 and C22:6, $n=2)$, and PCs (C38:6, C40:6, and C40:9, $n=3$ ) and lower levels of 11 metabolites including CEs (C14:0, C16:0, C18:0, C18: 2 , and C18:3, $n=5$ ), anthranilic acid, bilirubin, cytosine, 1-methylguanine, and 2 steroids (Table 2 and Additional file 1: Table S1). However, metabolomic differences were modest in women with MPTD only, who had lower levels of 4 PEs (C32:1, C34:2, C36:2, and C36:4) than term controls. In comparison, women with sPTD had significantly lower plasma levels of 13 metabolites (3 amino acids, 3 nucleotides, 2 steroids, C9 carnitine, anthranilic acid, bilirubin, 4-acetamidobutanoate, and trimethylamine- $N$-oxide) and higher levels of 3 metabolites (pyroglutamic acid, niacinamide, and phosphocholine), compared to the term controls. No significant associations were observed between SPTD and lipid metabolites. The identified associations remained largely unchanged when we further adjusted for the timing of maternal blood collection (postpartum day 1, 2, or 3 and above) in the regression models (data not shown).

We further examined the 47 metabolites that were associated with the group of $\mathrm{MPTD}$ and preeclampsia, compared to women with mPTD only. At $P<0.001$, women with both $\mathrm{MPTD}$ and preeclampsia had higher levels of four DAGs (C34:1, C34:2, C38:4, and C52:2) and two TAGs (C50:2 and C52:2) and lower levels of four CEs (C16:0, C18:0, C18:2, and C18:3) than women with $\mathrm{mPTD}$ only, suggesting specific associations with preeclampsia.

\section{Metabolite patterns related to PTD/preeclampsia subgroups by metabolomic network analyses}

With WGCNA network analysis, 329 metabolites were grouped into 8 metabolic network modules, annotated as different colors. Metabolites in each module are presented in Additional file 1: Table S2. Figure 2 presents the correlations between each PTD/preeclampsia subgroup and each module score. With the adjustment of covariates and with $P<0.006$ ( $=0.05 / 8$ modules) as the significance cutoff, we observed that women with $\mathrm{mPTD}$ and preeclampsia had significantly higher scores for the 
Table 1 Population characteristics of the 1330 women enrolled in the Boston Birth Cohort, stratified by preterm delivery subgroups

\begin{tabular}{|c|c|c|c|c|}
\hline \multirow[t]{2}{*}{ Variables } & \multirow{2}{*}{$\begin{array}{l}\text { Term } \\
\text { delivery }\end{array}$} & \multicolumn{3}{|c|}{ Preterm delivery (PTD) subgroups $^{\mathbf{a}}$} \\
\hline & & mPTD + preeclampsia & mPTD only & sPTD \\
\hline $\bar{N}$ & 980 & 79 & 52 & 219 \\
\hline Maternal age (years), $\mathrm{M} \pm \mathrm{SD}$ & $28.1 \pm 6.6$ & $31.1 \pm 6.5^{* *}$ & $30.2 \pm 7.2$ & $28.6 \pm 6.4$ \\
\hline \multicolumn{5}{|l|}{ Ethnicity } \\
\hline African American & $621(63.4)$ & $61(77.2)$ & $27(51.9)$ & $148(67.6)$ \\
\hline White & $40(4.1)$ & $2(2.5)$ & $2(3.9)$ & $12(5.5)$ \\
\hline Hispanic & $200(20.4)$ & $12(15.2)$ & $17(32.7)$ & $45(20.5)$ \\
\hline Others & $119(12.1)$ & $4(5.1)$ & $6(11.5)$ & $14(6.4)$ \\
\hline Maternal birthplace: non-US-born & $620(63.3)$ & $50(63.3)$ & $33(63.5)$ & $115(52.5)^{* *}$ \\
\hline Married & $338(34.5)$ & $26(32.9)$ & $17(32.7)$ & $54(24.7)^{*}$ \\
\hline \multicolumn{5}{|l|}{ Pregestational BMI $\left(\mathrm{kg} / \mathrm{m}^{2}\right)$ category } \\
\hline Normal & $470(48.0)$ & $21(26.6)^{* *}$ & $16(30.8)$ & $104(47.5)$ \\
\hline Overweight & $258(26.3)$ & $24(30.4)$ & $17(32.7)$ & $62(28.3)$ \\
\hline Obese & $208(21.2)$ & $32(40.5)$ & $16(30.8)$ & $43(19.6)$ \\
\hline Unknown & $44(4.5)$ & $2(2.5)$ & $3(5.8)$ & $10(4.6)$ \\
\hline \multicolumn{5}{|l|}{ Highest education level } \\
\hline$<$ High school & $273(27.9)$ & $12(15.2)$ & $21(40.4)$ & $62(28.3)$ \\
\hline High school & $362(36.9)$ & $39(49.4)$ & $18(34.6)$ & $86(39.3)$ \\
\hline College or above & $340(34.7)$ & $28(35.4)$ & $13(25.0)$ & $70(32.0)$ \\
\hline Missing & $5(0.5)$ & $0(0.0)$ & $0(0.0)$ & $1(0.5)$ \\
\hline Nulliparity & $430(43.9)$ & $34(43.0)$ & $17(32.7)$ & $97(44.3)$ \\
\hline \multicolumn{5}{|l|}{ Maternal smoking during pregnancy } \\
\hline Never & $840(85.7)$ & $62(78.5)$ & $41(78.8)$ & $161(73.5)^{* * *}$ \\
\hline Quitter & $64(6.5)$ & $8(10.1)$ & $3(5.8)$ & $20(9.1)$ \\
\hline Current & $64(6.5)$ & $8(10.1)$ & $8(15.4)$ & $38(17.4)$ \\
\hline Unknown & $12(1.2)$ & $1(1.3)$ & $0(0.0)$ & $0(0.0)$ \\
\hline Alcohol drinking during pregnancy & $73(7.4)$ & $3(3.8)$ & $5(9.6)$ & $20(9.1)$ \\
\hline \multicolumn{5}{|l|}{ Stress during lifetime } \\
\hline Mild & $410(41.8)$ & $29(36.7)^{*}$ & $16(30.8)$ & $76(34.7)$ \\
\hline Average & $490(50.0)$ & $36(45.6)$ & $30(57.7)$ & $118(53.9)$ \\
\hline Stressful & $80(8.2)$ & $14(17.7)$ & $6(11.5)$ & $25(11.4)$ \\
\hline Chronic hypertension & $37(3.8)$ & $28(35.9)^{* * *}$ & $6(11.5)^{*}$ & $12(5.5)$ \\
\hline Gestational diabetes & $69(7.0)$ & $9(11.4)$ & $4(7.7)$ & $16(7.3)$ \\
\hline Pregestational diabetes & $37(3.8)$ & $12(15.2)^{* * *}$ & $4(7.7)$ & $12(5.5)$ \\
\hline Illicit drug use during pregnancy & $66(6.7)$ & $9(11.4)$ & $6(11.5)$ & $34(15.5)^{* * *}$ \\
\hline Mode of delivery, c-section & $311(31.8)$ & $52(65.8)^{* * *}$ & $42(80.8)^{* * *}$ & $65(29.7)$ \\
\hline Infant's sex, male & $497(50.7)$ & $30(38.0)^{*}$ & 31 (59.6) & $123(56.2)$ \\
\hline
\end{tabular}

a $m P T D$, medically indicated PTD; the "mPTD + preeclampsia" group refers to women with mPTD and preeclampsia (including preeclampsia, eclampsia, and hemolysis, elevated liver enzymes, and low platelet syndrome (HELLP)); SPTD, spontaneous preterm delivery

${ }^{*} P<0.05 ;{ }^{* *} P<0.01 ;{ }^{* *} P<0.001$ for the difference of population characteristics in women of different PTD subgroups compared to term controls, via ANOVA tests and chi-squared tests for continuous and categorical variables, respectively

"yellow" (C16:0 CE as the top hub metabolite that was inversely correlated with the module score, $P=7.8 \times$ $10^{-7}$ ), "black" (C14:1 carnitine as the top hub metabolite, $P=1.6 \times 10^{-5}$ ), and "green" modules (pseudouridine as the top hub metabolite, $P=0.0007$ ) than term controls
(Table 3$)$. Women with mPTD only had a significantly lower score of the "blue" module $(P=0.004)$, while women with SPTD had a significantly lower score of the "green" module $(P=0.003$, Table 3$)$, compared to term controls. 

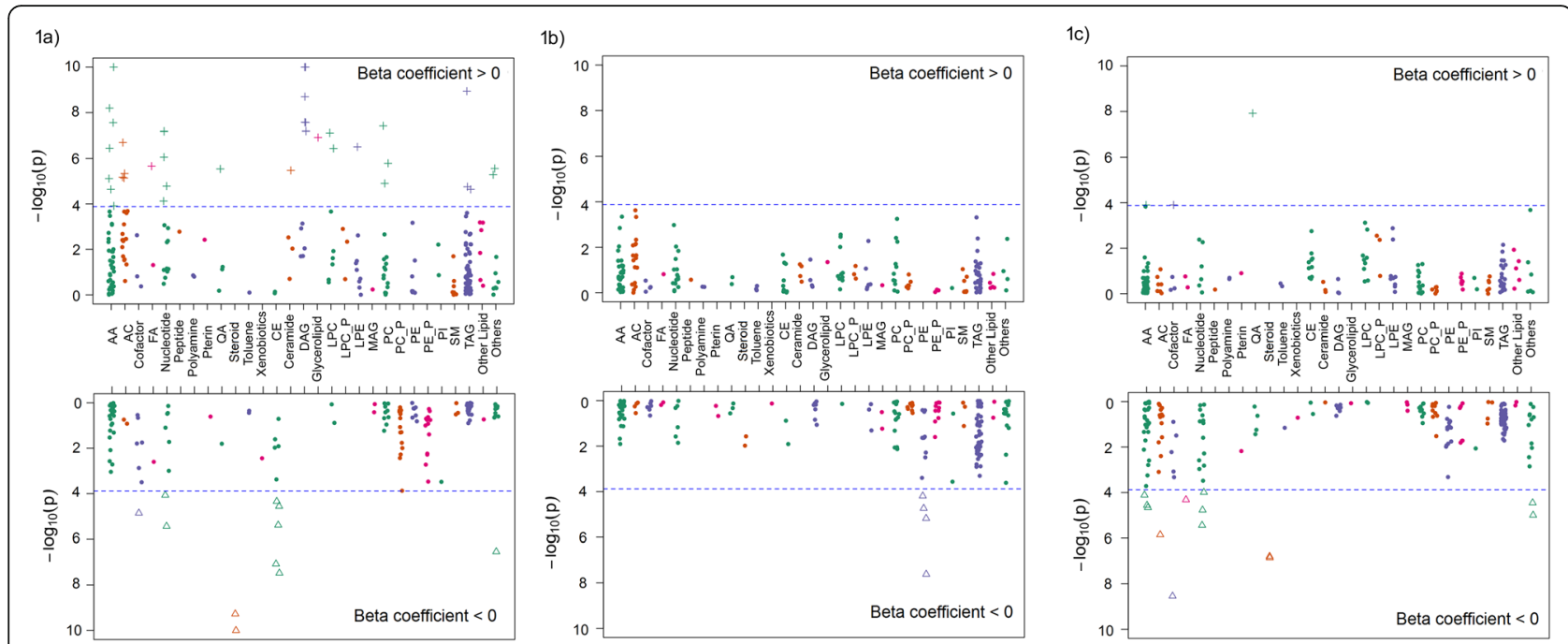

Fig. 1 Manhattan plot for the metabolomic differences in women with each preterm delivery (PTD) subgroup compared to women with term delivery. The upper panel presents metabolites that were higher, and the lower panel presents metabolites that were lower in women with medically indicated PTD and preeclampsia (a), in women with medically indicated PTD only (b), and in women with spontaneous PTD (c), respectively. The analyses were adjusted for maternal age at delivery, maternal ethnicity/race, maternal birthplace, pregestational BMI category, pregestational diabetes, chronic hypertension, marital status, highest education level, parity, smoking during pregnancy, illicit drug use, lifetime stress, and fetal sex. X-axis represents the metabolite classes. AA amino acid, AC acylcarnitine, FA fatty acid and conjugate, QA quaternary amine, CE cholesterol ester, DAG diacylglycerol, LPC lysophosphatidylcholine, LPC_P lysophosphatidylcholine plasmalogen, LPE lysophosphatidylethanolamine, MAG monoacylglycerol, $P C$ phosphatidylcholine, $P C \_P$ phosphatidylcholine plasmalogen, $P E$ phosphatidylethanolamine, $P E_{\_} P$ phosphatidylethanolamine plasmalogen, PI phosphatidylinositol, SM sphingomyelin, TAG triacylglycerol

Stratified analyses were further performed by maternal ethnicity and maternal parity. As shown in Additional file 1: Table S3 and Table S4, the associations between the PTD/preeclampsia subgroups and each metabolite module were largely comparable between Black and non-Black mothers and between nulliparity and multiparity mothers.

\section{Exploratory analyses of early and late SPTD}

When women with sPTD were split into early $(<33$ weeks of gestation, $n=67)$ and late sPTD $\left(33-36^{6 / 7}\right.$ weeks of gestation, $n=152$ ), and compared to term controls, we observed that the identified sPTD-associated differences in 16 individual metabolites were predominantly observed in women with early SPTD rather than in women with late SPTD (Additional file 1: Table S5). In addition, another 20 metabolites were significantly different in women with early sPTD, including 6 lipids (4 CEs and 2 LPCs) that were higher and 4 lipids (3 PEs and C36:2 phosphatidylinositol) that were lower in mothers with early sPTD (Additional file 1: Table S5 and Additional file 2: Fig. S1). Of note, the identified two LPCs (C22:5 and C22:6) were also significantly higher in women with MPTD and preeclampsia, and two PEs (C34:2 and C36:2) that were lower in women with early sPTD were also significantly lower in women with mPTD only. In the WGCNA analyses, women with early sPTD had significantly lower scores of the "yellow" (C16: $0 \mathrm{CE}$ as the top hub metabolite) and "green" (pseudouridine as the top hub metabolite) modules than term controls (Additional file 1: Table S6).

\section{Discussion}

To our knowledge, this study represents the largest to date to explore postpartum metabolomic profiles significantly associated with PTD/preeclampsia subgroups from a highrisk US multi-ethnic population. A total of 47, 4, and 16 metabolites were significantly different in women with mPTD and preeclampsia, in women with MPTD only, and in women with SPTD, respectively, compared with term controls. The broad-scale metabolite differences in women with MPTD and preeclampsia predominantly involve acylcarnitines and lipids. The neutral lipids, including DAGs, TAGs, and CEs, were significantly higher in women with mPTD and preeclampsia than in women with MPTD only, suggesting potential specific associations with preeclampsia. This finding is in line with clinically observed significant and independent adverse impact of preeclampsia-related PTD on maternal cardiometabolic health $[1,3]$. We also observed significant lipid metabolite alteration in mothers with early sPTD, including those lipids (LPCs and PEs) that were associated with medically indicated PTD, indicating shared pathways among different PTD subtypes in affecting maternal metabolic health. While a comprehensive discussion on the differences of all the metabolites identified in this study is beyond the word limit of this report, below we just highlight the major findings and relevant literature. 
Table 2 Maternal metabolites that were significantly different in women of each preterm delivery (PTD) subgroups, compared to women with term delivery

\begin{tabular}{|c|c|c|c|c|c|c|c|}
\hline \multirow[t]{2}{*}{ Maternal metabolites $^{\mathbf{a}}$} & \multirow[t]{2}{*}{ Metabolite classes } & \multicolumn{2}{|c|}{ mPTD + preeclampsiab } & \multicolumn{2}{|l|}{ mPTD only } & \multicolumn{2}{|l|}{ sPTD } \\
\hline & & $\overline{B e t a} \pm \mathrm{SE}^{\mathrm{C}}$ & $P$ & Beta $\pm \mathrm{SE}^{\mathrm{C}}$ & $P$ & Beta $\pm S E^{c}$ & $P$ \\
\hline Alloisoleucine & Amino acid & $-0.02 \pm 0.12$ & 0.88 & $-0.32 \pm 0.14$ & 0.02 & $-0.31 \pm 0.07$ & $2.2 \times 10^{-5}$ \\
\hline Creatine & Amino acid & $0.98 \pm 0.12$ & $1.4 \times 10^{-15}$ & $0.34 \pm 0.14$ & 0.01 & $0.28 \pm 0.07$ & $1.5 \times 10^{-4}$ \\
\hline Creatinine & Amino acid & $0.27 \pm 0.12$ & 0.02 & $-0.03 \pm 0.14$ & 0.82 & $-0.29 \pm 0.07$ & $7.8 \times 10^{-5}$ \\
\hline Cystine & Amino acid & $0.70 \pm 0.12$ & $6.4 \times 10^{-9}$ & $0.08 \pm 0.14$ & 0.56 & $0.02 \pm 0.07$ & 0.82 \\
\hline Glutamine & Amino acid & $0.52 \pm 0.12$ & $2.3 \times 10^{-5}$ & $0.21 \pm 0.14$ & 0.13 & $0.01 \pm 0.07$ & 0.88 \\
\hline Homocitrulline & Amino acid & $0.47 \pm 0.12$ & $1.2 \times 10^{-4}$ & $-0.03 \pm 0.14$ & 0.84 & $-0.09 \pm 0.07$ & 0.22 \\
\hline N-Acetyltryptophan & Amino acid & $0.68 \pm 0.12$ & $2.8 \times 10^{-8}$ & $-0.10 \pm 0.14$ & 0.47 & $-0.10 \pm 0.07$ & 0.19 \\
\hline N-Acetylputrescine & Amino acid & $0.55 \pm 0.12$ & $7.7 \times 10^{-6}$ & $0.22 \pm 0.14$ & 0.12 & $-0.13 \pm 0.07$ & 0.07 \\
\hline N6,N6,N6-trimethyllysine & Amino acid & $0.62 \pm 0.12$ & $3.5 \times 10^{-7}$ & $0.29 \pm 0.14$ & 0.04 & $0.08 \pm 0.07$ & 0.31 \\
\hline Pyroglutamic acid & Amino acid & $0.31 \pm 0.12$ & 0.01 & $0.18 \pm 0.14$ & 0.22 & $0.29 \pm 0.08$ & $1.3 \times 10^{-4}$ \\
\hline C3 carnitine & Acylcarnitine & $0.57 \pm 0.12$ & $4.6 \times 10^{-6}$ & $0.09 \pm 0.14$ & 0.54 & $0.13 \pm 0.08$ & 0.08 \\
\hline C5-DC carnitine & Acylcarnitine & $0.63 \pm 0.12$ & $2.0 \times 10^{-7}$ & $0.13 \pm 0.14$ & 0.37 & $-0.09 \pm 0.07$ & 0.21 \\
\hline C8 carnitine & Acylcarnitine & $0.54 \pm 0.12$ & $7.3 \times 10^{-6}$ & $0.30 \pm 0.14$ & 0.03 & $-0.05 \pm 0.07$ & 0.52 \\
\hline C9 carnitine & Acylcarnitine & $-0.16 \pm 0.12$ & 0.18 & $-0.15 \pm 0.14$ & 0.28 & $-0.36 \pm 0.07$ & $1.4 \times 10^{-6}$ \\
\hline Anthranilic acid & Aminobenzoic acid & $-0.63 \pm 0.12$ & $2.9 \times 10^{-7}$ & $-0.52 \pm 0.14$ & $2.4 \times 10^{-4}$ & $-0.33 \pm 0.08$ & $1.0 \times 10^{-5}$ \\
\hline Bilirubin & Cofactors & $-0.52 \pm 0.12$ & $1.4 \times 10^{-5}$ & $0.15 \pm 0.14$ & 0.29 & $-0.44 \pm 0.07$ & $2.9 \times 10^{-9}$ \\
\hline Niacinamide & Cofactors & $0.18 \pm 0.12$ & 0.15 & $0.07 \pm 0.14$ & 0.60 & $0.29 \pm 0.08$ & $1.2 \times 10^{-4}$ \\
\hline 4-Acetamidobutanoate & Fatty acid & $0.57 \pm 0.12$ & $2.2 \times 10^{-6}$ & $-0.03 \pm 0.14$ & 0.84 & $-0.30 \pm 0.07$ & $4.8 \times 10^{-5}$ \\
\hline Imidazole propionate & Imidazole & $0.56 \pm 0.12$ & $2.8 \times 10^{-6}$ & $0.39 \pm 0.14$ & 0.004 & $0.27 \pm 0.07$ & $2.1 \times 10^{-4}$ \\
\hline Cytosine & Nucleotide & $-0.56 \pm 0.12$ & $3.7 \times 10^{-6}$ & $0.16 \pm 0.14$ & 0.25 & $-0.21 \pm 0.07$ & 0.01 \\
\hline 6,8-Dihydroxypurine & Nucleotide & $0.60 \pm 0.12$ & $8.8 \times 10^{-7}$ & $0.24 \pm 0.14$ & 0.09 & $0.09 \pm 0.07$ & 0.23 \\
\hline 1-Methyladenosine & Nucleotide & $-0.04 \pm 0.12$ & 0.72 & $-0.00 \pm 0.14$ & 0.97 & $-0.29 \pm 0.07$ & $1.0 \times 10^{-4}$ \\
\hline 1-Methylguanine & Nucleotide & $-0.48 \pm 0.12$ & $8.6 \times 10^{-5}$ & $0.04 \pm 0.14$ & 0.77 & $-0.23 \pm 0.08$ & 0.003 \\
\hline 7-Methylguanine & Nucleotide & $-0.29 \pm 0.12$ & 0.02 & $-0.10 \pm 0.14$ & 0.48 & $-0.35 \pm 0.07$ & $3.6 \times 10^{-6}$ \\
\hline Urate & Nucleotide & $0.65 \pm 0.12$ & $6.5 \times 10^{-8}$ & $0.29 \pm 0.14$ & 0.03 & $-0.31 \pm 0.07$ & $1.7 \times 10^{-5}$ \\
\hline Carnitine & Quaternary amine & $0.58 \pm 0.12$ & $2.9 \times 10^{-6}$ & $0.12 \pm 0.14$ & 0.42 & $-0.09 \pm 0.08$ & 0.24 \\
\hline Phosphocholine & Quaternary amine & $0.23 \pm 0.12$ & 0.06 & $0.18 \pm 0.14$ & 0.21 & $0.43 \pm 0.08$ & $1.21 \times 10^{-8}$ \\
\hline Cortisol & Steroid & $-0.95 \pm 0.12$ & $3.1 \times 10^{-15}$ & $-0.35 \pm 0.14$ & 0.01 & $-0.39 \pm 0.07$ & $1.61 \times 10^{-7}$ \\
\hline \multicolumn{8}{|l|}{ Lipids } \\
\hline C14:0 CE & Cholesteryl ester & $-0.52 \pm 0.11$ & $4.2 \times 10^{-6}$ & $-0.33 \pm 0.13$ & 0.01 & $-0.07 \pm 0.07$ & 0.28 \\
\hline C18:2 CE & Cholesteryl ester & $-0.66 \pm 0.12$ & $3.4 \times 10^{-8}$ & $0.00 \pm 0.14$ & 0.98 & $0.09 \pm 0.07$ & 0.22 \\
\hline C16:0 Ceramide & Ceramide & $0.55 \pm 0.12$ & $3.4 \times 10^{-6}$ & $0.26 \pm 0.14$ & 0.06 & $0.07 \pm 0.07$ & 0.30 \\
\hline C34:1 DAG/TAG fragment & Diacylglycerol & $0.64 \pm 0.12$ & $1.2 \times 10^{-7}$ & $0.28 \pm 0.14$ & 0.04 & $-0.01 \pm 0.07$ & 0.85 \\
\hline C36:2 DAG & Diacylglycerol & $0.78 \pm 0.12$ & $4.4 \times 10^{-11}$ & $0.10 \pm 0.13$ & 0.47 & $-0.05 \pm 0.07$ & 0.47 \\
\hline C38:5 DAG & Diacylglycerol & $0.79 \pm 0.12$ & $4.5 \times 10^{-11}$ & $0.15 \pm 0.14$ & 0.28 & $0.09 \pm 0.07$ & 0.23 \\
\hline C22:6 LPC & LPC & $0.65 \pm 0.12$ & $7.7 \times 10^{-8}$ & $0.41 \pm 0.14$ & 0.003 & $0.25 \pm 0.07$ & $7.6 \times 10^{-4}$ \\
\hline C22:6 LPE & LPE & $0.62 \pm 0.12$ & $3.1 \times 10^{-7}$ & $0.39 \pm 0.14$ & 0.01 & $0.24 \pm 0.07$ & 0.001 \\
\hline$C 40: 6$ PC & PC & $0.65 \pm 0.12$ & $3.8 \times 10^{-8}$ & $0.38 \pm 0.14$ & 0.01 & $0.12 \pm 0.07$ & 0.10 \\
\hline C34:2 PE & PE & $-0.06 \pm 0.11$ & 0.62 & $-0.74 \pm 0.13$ & $2.4 \times 10^{-8}$ & $-0.25 \pm 0.07$ & $4.8 \times 10^{-4}$ \\
\hline C52:2 TAG & Triacylglycerol & $0.71 \pm 0.12$ & $1.1 \times 10^{-9}$ & $0.06 \pm 0.13$ & 0.63 & $-0.15 \pm 0.07$ & 0.04 \\
\hline
\end{tabular}


Table 2 Maternal metabolites that were significantly different in women of each preterm delivery (PTD) subgroups, compared to women with term delivery (Continued)

\begin{tabular}{|c|c|c|c|c|c|c|c|}
\hline \multirow[t]{2}{*}{ Maternal metabolites $^{\mathbf{a}}$} & \multirow[t]{2}{*}{ Metabolite classes } & \multicolumn{2}{|c|}{ mPTD + preeclampsia ${ }^{\mathbf{b}}$} & \multicolumn{2}{|l|}{ mPTD only } & \multicolumn{2}{|l|}{ sPTD } \\
\hline & & $\overline{B e t a} \pm \mathrm{SE}^{\mathrm{C}}$ & $P$ & $\overline{B e t a} \pm \mathrm{SE}^{\mathrm{C}}$ & $P$ & $\overline{B e t a} \pm \mathrm{SE}^{\mathrm{C}}$ & $P$ \\
\hline \multicolumn{8}{|l|}{ Others } \\
\hline Methylguanidine & Others & $0.56 \pm 0.12$ & $5.2 \times 10^{-6}$ & $-0.17 \pm 0.14$ & 0.23 & $-0.10 \pm 0.07$ & 0.16 \\
\hline Trimethylamine- $\mathrm{N}$-oxide & Others & $-0.05 \pm 0.12$ & 0.70 & $0.04 \pm 0.14$ & 0.78 & $-0.31 \pm 0.07$ & $3.5 \times 10^{-5}$ \\
\hline
\end{tabular}

\section{Differences in acylcarnitine associated with MPTD and preeclampsia}

Significantly higher levels of multiple acylcarnitines (metabolites related to cellular energy production) were mainly observed in women with mPTD and preeclampsia. Acylcarnitines are responsible for the transport of longchain fatty acids from the cytoplasm into the mitochondria for $\beta$-oxidation. Fatty acids play an important role during pregnancy as metabolic fuel for the placenta [24]. Elevated acylcarnitines have been reported as potential biomarkers for preeclampsia [25-27]. Using the network analyses via WGCNA, acylcarnitines together with metabolites in purine metabolism (6,8-dihydroxypurine, xanthine, and xanthosine), which all were higher in women with $\mathrm{mPTD}$ and preeclampsia, were classified into the "black" module, suggesting coexpression of these metabolites.

\section{Alterations in lipid metabolites among PTD/preeclampsia subgroups}

Neutral lipids, including DAGs, TAGs, and CEs, constitute the most abundant group of lipids and serve as energy and carbon storage. These lipids, or the score for the "yellow" module, were significantly altered in women with $\mathrm{MPTD}$ and preeclampsia compared to women with mPTD only, suggesting preeclampsia-specific associations. Accumulation of DAGs has been reported to play a role in insulin resistance in cell [28]. DAGs are precursors to TAGs which play an important role in the storage of energy and fatty acids. In our study, the major TAGs that were higher in women with MPTD and preeclampsia included TAG C50:2, C52:2, and C52:3. These TAGs are of short- or medium-carbon chains which are likely hydrolyzed to release saturated (i.e., C16:0 palmitic acid or C18:0 stearic acid) and monounsaturated (i.e.,

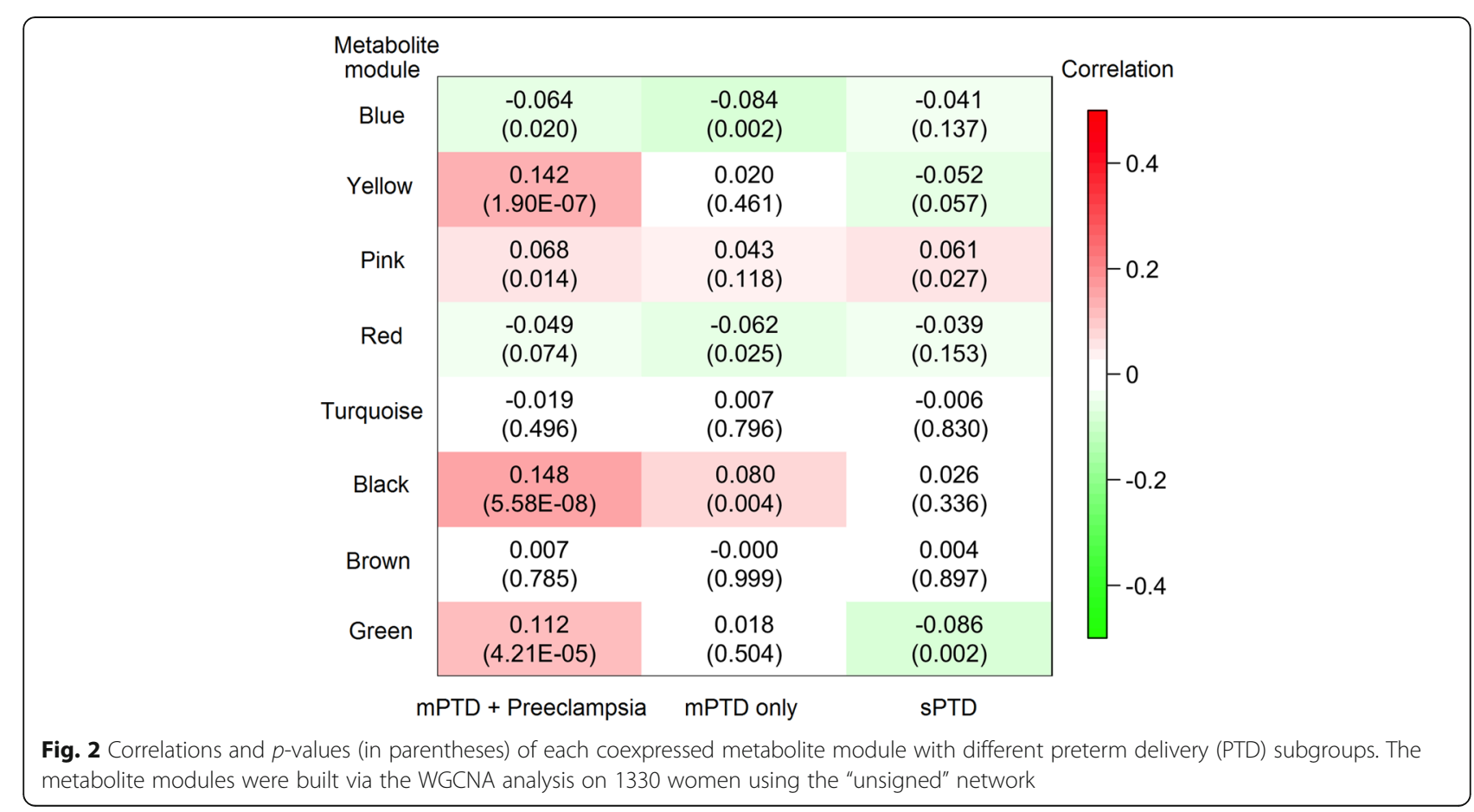


Table 3 Associations of the eight metabolite modules with each preterm delivery (PTD) subgroup, compared to women with term delivery

\begin{tabular}{|c|c|c|c|c|c|c|c|c|}
\hline \multirow[t]{2}{*}{ Module $^{a}$} & \multirow[t]{2}{*}{$N_{\text {metabolite }}$} & \multirow[t]{2}{*}{ Top hub metabolite ${ }^{b}$} & \multicolumn{2}{|c|}{ mPTD + preeclampsia } & \multicolumn{2}{|l|}{ mPTD only } & \multicolumn{2}{|l|}{ sPTD } \\
\hline & & & $\overline{B e t a} \pm \mathrm{SE}^{\mathrm{c}}$ & $P$ & $\overline{B e t a} \pm \mathrm{SE}^{\mathrm{C}}$ & $P$ & $\overline{B e t a} \pm \mathrm{SE}^{\mathrm{c}}$ & $P$ \\
\hline Blue & 54 & C44:1 TAG & $0.000 \pm 0.003$ & 0.98 & $-0.011 \pm 0.004$ & $0.004^{*}$ & $-0.003 \pm 0.002$ & 0.10 \\
\hline Yellow & 38 & C16:0 CE or C36:2 DAG & $0.016 \pm 0.003$ & $7.8 \times 10^{-7 *}$ & $-0.001 \pm 0.004$ & 0.86 & $-0.004 \pm 0.002$ & 0.06 \\
\hline Pink & 26 & C18:1 LPC & $0.009 \pm 0.003$ & 0.007 & $0.005 \pm 0.004$ & 0.24 & $0.005 \pm 0.002$ & 0.03 \\
\hline Red & 36 & C32:2 PC & $0.001 \pm 0.003$ & 0.76 & $-0.008 \pm 0.004$ & 0.03 & $-0.002 \pm 0.002$ & 0.30 \\
\hline Turquoise & 63 & C16:0 sphingomyelin & $-0.001 \pm 0.003$ & 0.74 & $0.001 \pm 0.004$ & 0.71 & $0.000 \pm 0.002$ & 0.85 \\
\hline Black & 34 & C14:1 carnitine & $0.015 \pm 0.003$ & $1.6 \times 10^{-5 *}$ & $0.009 \pm 0.004$ & 0.02 & $0.002 \pm 0.002$ & 0.33 \\
\hline Brown & 41 & Valine & $0.003 \pm 0.003$ & 0.38 & $0.001 \pm 0.004$ & 0.77 & $0.001 \pm 0.002$ & 0.66 \\
\hline Green & 37 & Pseudouridine & $0.011 \pm 0.003$ & $7.0 \times 10^{-4 *}$ & $0.002 \pm 0.004$ & 0.54 & $-0.006 \pm 0.002$ & $0.003^{*}$ \\
\hline
\end{tabular}

mPTD medically indicated PTD, CE cholesteryl ester, DAG diacylglycerol, LPC lysophosphatidylcholine, PC phosphatidylcholine, TAG triacylglycerol

aThe WGCNA analysis was conducted on 1330 samples using the "unsigned" network, where the highly correlated (either positive or negative) metabolites were grouped into the same module

${ }^{\mathrm{b}}$ The top hub metabolite was defined as the one with the highest mean correlation with the other metabolites in the same module

${ }^{c}$ Adjusted for maternal age at delivery, maternal ethnicity/race, maternal birthplace, pregestational BMI category, pregestational diabetes, chronic hypertension,

marital status, highest education level, parity, smoking during pregnancy, illicit drug use, lifetime stress, and fetal sex

${ }^{\mathrm{d}} \mathrm{C} 16: 0 \mathrm{CE}$ is negatively correlated with the "yellow" module score while C36:2 DAG is positively correlated with the "yellow" module score

*Significant after Bonferroni corrections for multiple testing on 8 metabolite modules

C18:1 oleic acid) fatty acids. Oxidation of saturated and monounsaturated fatty acids provides more energy than oxidation of polyunsaturated fatty acids of the same length [29]. Accumulation of such short- or mediumcarbon chain TAGs may indicate dysfunctional energy metabolism and may induce pro-inflammatory signaling [30], which has been proposed as a feature of multiple PTD-related diseases, including diabetes $[22,31]$ and CVD [14]. Another type of neutral lipid, CEs, was inversely correlated with DAGs and TAGs, which may partly explain the lower levels of CEs (C14:0, C16:0, C18:0, C18:2, and C18:3) that we observed in women with $\mathrm{mPTD}$ and preeclampsia. Consistently, lower CEs have been linked to unhealthy lifestyles including a lack of physical activity (including C16:0 and C18:2) [32] and an insulinemic dietary pattern (including C14:0 and C18: 3) [33]. However, the relationships between CEs and CVD risk have been inconclusive, including both positive [14] and inverse associations [34, 35]. In comparison, we observed that multiple CEs (C18:0, C20:3, C20:4, and C22:4) were positively associated with early sPTD and that the score for the "yellow" module was inversely associated with early SPTD in this study. Future studies are needed to explore whether and how differences in levels of such neutral lipids may explain the adverse impact of preeclampsia-related PTD and early SPTD on CVD risk.

\section{Altered metabolites of amino acids and nucleotides by different PTD/preeclampsia subgroups}

Different levels of amino acids and nucleotides were observed in women with mPTD and preeclampsia and in women with sPTD (especially those with early sPTD), including both shared and unique metabolites. For example, anthranilic acid and bilirubin were lower across the different PTD subtypes. Anthranilic acid is the metabolite of tryptophan. Tryptophan level during pregnancy was lower in mothers with PTD [36]. Anthranilic acid derivatives have been reported to have antiinflammatory function [37, 38]. Collectively, this evidence may help to explain the inverse relationship between PTD and anthranilic acid. Bilirubin is a bile pigment with potent antioxidant properties that inhibit lipid oxidation and atherosclerotic development $[39,40]$. A number of studies have reported an inverse association between bilirubin levels and risk of hypertension, diabetes, and CVD [41, 42]. Taken together, these findings suggest the hypothesis that inflammation and elevated oxidative stress are potential mechanisms via which both preeclampsia-related MPTD and early sPTD may influence future cardiometabolic health.

The main strengths of our study included a large sample size, a well-validated metabolomic platform and detailed information on covariates, and more importantly, our ability to define PTD/preeclampsia subgroups, which are clinically and pathogenically important to gain new insight. Some limitations of our study should also be acknowledged. First, the BBC was particularly designed to study preterm delivery in an inner-city, predominantly minority population; therefore, caution is needed when generalizing our findings to other populations. Second, there is lack of a replication cohort in this study. We cannot exclude the possibility that some of these findings may not be replicated in another cohort. Third, maternal metabolites were measured in plasma collected within 1-3 days after delivery, which was at a time of significant flux with respect to molecules in the circulation. Future studies are needed to investigate whether 
the identified metabolite differences (especially those associated with $\mathrm{mPTD}$ and preeclampsia) are persistent across different time periods postpartum and whether they are associated with future CVD risk. Fourth, we could not distinguish early-onset versus late-onset preeclampsia in this study. Given the possibility that prolonged exposure to preeclampsia may be associated with an increased risk of CVD, further studies are needed to test whether early-onset preeclampsia and late-onset preeclampsia have different associations with maternal postpartum metabolomic profiles. Fifth, pregestational cardiovascular risk factors are important risk factors of gestational complications including preeclampsia [4345] and they may also lead to metabolomic differences postpartum. To minimize potential confounding effects, we adjusted for pregestational overweight/obesity, hypertension, and diabetes in our analyses. Pregestational dyslipidemia is another potential risk factor of preeclampsia $[44,45]$. However, information on pregestational dyslipidemia was not available in this study. Future studies are needed to assess potential confounding by pregestational dyslipidemia. Finally, we were only able to further stratify preterm delivery into early vs. late for the sPTD group, but not for other $\mathrm{mPTD} /$ preeclampsia subgroups due to limited sample size.

\section{Conclusion}

We demonstrated individual and clusters of maternal postpartum metabolites that were significantly and specifically associated with clinically observed subgroups: mPTD and preeclampsia, MPTD only, and SPTD, as well as some metabolites that were shared among the subgroups. Some of the identified lipid metabolites and/or related pathways, especially those associated with the MPTD and preeclampsia subgroup, have been reported as early predictors of future risk of CVD. Our study represents an early attempt to better understand metabolomic underpinnings of $\mathrm{PTD} /$ preeclampsia. Additional studies are warranted to confirm our findings and further investigate the link of these identified metabolites in mediating or modifying a woman's future cardiometabolic risk. A better understanding of the pathways from $\mathrm{PTD} /$ preeclampsia, postpartum metabolomic profiles, to maternal future cardiometabolic risk may lead to new risk assessment and intervention strategies and help to identify at-risk women early in the disease process when intervention may delay or prevent the onset of cardiometabolic disease.

\section{Supplementary information}

Supplementary information accompanies this paper at https://doi.org/10. 1186/s12916-020-01741-4.

Additional file 1: Table S1. Other maternal metabolites that were significantly different in women of each preterm delivery (PTD) subgroup, compared to women of term delivery. Table S2. Individual metabolites included in each of the 8 metabolite modules. Table S3. Associations of metabolite modules with different preterm delivery (PTD) subgroups, stratified by maternal parity. Table S4. Associations of metabolite modules with different preterm delivery (PTD) subgroups, stratified by maternal race / ethnicity. Table S5. Maternal metabolites that were significantly different in women with late SPTD and in women with early SPTD, separately, compared to women with term delivery. Table S6. Associations of metabolite modules with early and late SPTD, separately, compared to women with term delivery.

Additional file 2: Figure S1. Manhattan plot for the metabolomic differences in women with early and late spontaneous PTD, separately, compared to women with term delivery.

\section{Abbreviations}

BBC: Boston Birth Cohort; BMI: Body mass index; CE: Cholesteryl ester; CV: Coefficient of variance; CVD: Cardiovascular disease; DAG: Diacylglycerol; EMR: Electronic medical record; LPC: Lysophosphatidylcholine;

LPE: Lysophosphatidylethanolamine; MPTD: Medically indicated PTD; PC: Phosphatidylcholine; PE: Phosphatidylethanolamine;

PI: Phosphatidylinositol; PTD: Preterm delivery; sPTD: Spontaneous PTD;

TAG: Triacylglycerol; WGCNA: Weighted gene coexpression network analysis

\section{Acknowledgements}

The authors wish to thank the study participants in the BBC, the nursing staff at labor and delivery of the Boston Medical Center, and the field team for their contributions to the Boston Birth Cohort. Linda Rosen in the Clinical Data Warehouse assisted in obtaining relevant clinical data from electronic medical records. The Clinical Data Warehouse service is supported by Boston University's Clinical and Translational Institute and the National Institutes of Health Clinical and Translational Science awards (grant U54-TR001012). We thank Tami Bartell for her scientific editing of the manuscript text. We thank Dr. Meir Stampfer for his careful review and very helpful edits and comments.

\section{Authors' contributions}

$\mathrm{XH}, \mathrm{FH}$, and $\mathrm{XW}$ were responsible for the study concept and design. $\mathrm{XH}, \mathrm{LL}$, and XW were responsible for drafting the manuscript. CC supervised laboratory metabolomic assays and quality control. $X H, B Y Z, Y J$, and $L L$ assumed major responsibilities for statistical analyses and data presentations. GW and YJ prepared blood samples for the lab assay. XW was responsible for overseeing the acquisition of the epidemiological and clinical data as well as biospecimens. All the authors were responsible for the critical review and revision of the manuscript and contributed to data interpretations. $\mathrm{XH}$ and XW have full access to all the data in the study and take responsibility for the integrity of the data and the accuracy of the data analysis. All authors read and approved the final manuscript.

\section{Funding}

The Boston Birth Cohort (the parent study, PI, Wang X) was supported in part by the March of Dimes PERI grants (20-FY02-56, \#21-FY07-605), the Health Resources and Services Administration (HRSA) of the U.S. Department of Health and Human Services (HHS) under grant number R40MC27443, Autism Field-initiated Innovative Research Studies Program; and grant number UJ2MC31074, Autism Single Investigator Innovation Program; and the National Institutes of Health (NIH) grants (R21ES011666, R01HD041702, R21HD066471, U01Al090727, R21Al079872, R01HD086013, R01HD098232, R21AI154233 and R01ES031272). Dr. Hong is also supported by NICHD (PI: Hong X, R03HD096136) and Hopkins Population Center (NICHD

R24HD042854). This information or content and conclusions are those of the author and should not be construed as the official position or policy of, nor should any endorsements be inferred by HRSA, HHS, or the US Government.

\section{Availability of data and materials}

The datasets used and/or analyzed for the current study are available from the corresponding author on reasonable request, and after the Institutional Review Board review and approval. 


\section{Ethics approval and consent to participate}

The study has been approved by the Institutional Review Boards of Boston Medical Center and Johns Hopkins Bloomberg School of Public Health. Written informed consent was obtained from each participant.

\section{Consent for publication}

Not applicable.

\section{Competing interests}

The authors declare that they have no competing interests.

\section{Author details}

${ }^{1}$ Center on the Early Life Origins of Disease, Department of Population, Family and Reproductive Health, Johns Hopkins University Bloomberg School of Public Health, 615 N. Wolfe St, Baltimore, MD 21205-2179, USA. ${ }^{2}$ Department of Biostatistics, Johns Hopkins University Bloomberg School of Public Health, 615 N. Wolfe St, Baltimore, MD 21205, USA. ${ }^{3}$ Department of Epidemiology, Harvard T.H. Chan School of Public Health, Boston, MA, USA. ${ }^{4}$ Department of Biostatistics, Harvard T.H. Chan School of Public Health, Boston, MA, USA. ${ }^{5}$ Broad Institute of MIT and Harvard University, Cambridge, MA, USA. ${ }^{6}$ Integrated Research Center for Fetal Medicine, Department of Gynecology and Obstetrics, Johns Hopkins University School of Medicine, Baltimore, MD 21287, USA. 'Department of Pediatrics, Boston University School of Medicine and Boston Medical Center, Boston, MA 02118, USA. ${ }^{8}$ Department of Nutrition, Harvard T.H. Chan School of Public Health, Boston, MA, USA. ${ }^{9}$ Channing Division of Network Medicine, Department of Medicine, Brigham and Women's Hospital and Harvard Medical School, Boston, MA, USA. ${ }^{10}$ Division of General Pediatrics \& Adolescent Medicine, Department of Pediatrics, Johns Hopkins University School of Medicine, Baltimore, MD 21205, USA.

Received: 7 May 2020 Accepted: 10 August 2020

Published online: 13 October 2020

\section{References}

1. Auger N, Potter BJ, He S, Healy-Profitos J, Schnitzer ME, Paradis G. Maternal cardiovascular disease 3 decades after preterm birth: longitudinal cohort study of pregnancy vascular disorders. Hypertension. 2020;75(3):788-795.

2. Bonamy AK, Parikh NI, Cnattingius S, Ludvigsson JF, Ingelsson E. Birth characteristics and subsequent risks of maternal cardiovascular disease: effects of gestational age and fetal growth. Circulation. 2011;124(25): 2839-46.

3. Tanz LJ, Stuart JJ, Williams PL, Rimm EB, Missmer SA, Rexrode KM, Mukamal KJ, Rich-Edwards JW. Preterm delivery and maternal cardiovascular disease in young and middle-aged adult women. Circulation. 2017;135(6):578-89.

4. Wu P, Gulati M, Kwok CS, Wong CW, Narain A, O'Brien S, Chew-Graham CA, Verma G, Kadam UT, Mamas MA. Preterm delivery and future risk of maternal cardiovascular disease: a systematic review and meta-analysis. $J$ Am Heart Assoc. 2018;7(2):e007809.

5. Murphy SL, Xu J, Kochanek KD, Curtin SC, Arias E. Deaths: final data for 2015. Natl Vital Stat Rep. 2017;66(6):1-75.

6. Minissian MB, Kilpatrick S, Eastwood JA, Robbins WA, Accortt EE, Wei J, Shufelt CL, Doering LV, Merz CNB. Association of spontaneous preterm delivery and future maternal cardiovascular disease. Circulation. 2018;137(8): $865-71$

7. Catov JM, Wu CS, Olsen J, Sutton-Tyrrell K, Li J, Nohr EA. Early or recurrent preterm birth and maternal cardiovascular disease risk. Ann Epidemiol. 2010; 20(8):604-9.

8. Zheng Y, Yu B, Alexander D, Steffen LM, Nettleton JA, Boerwinkle E. Metabolomic patterns and alcohol consumption in African Americans in the Atherosclerosis Risk in Communities Study. Am J Clin Nutr. 2014;99(6):1470-8.

9. Magnusson M, Lewis GD, Ericson U, Orho-Melander M, Hedblad B, Engstrom G, Ostling G, Clish C, Wang TJ, Gerszten RE, et al. A diabetes-predictive amino acid score and future cardiovascular disease. Eur Heart J. 2013;34(26): 1982-9.

10. Cheng S, Rhee EP, Larson MG, Lewis GD, McCabe EL, Shen D, Palma MJ, Roberts LD, Dejam A, Souza AL, et al. Metabolite profiling identifies pathways associated with metabolic risk in humans. Circulation. 2012; 125(18):2222-31.

11. Shah SH, Sun JL, Stevens RD, Bain JR, Muehlbauer MJ, Pieper KS, Haynes C, Hauser ER, Kraus WE, Granger CB, et al. Baseline metabolomic profiles predict cardiovascular events in patients at risk for coronary artery disease. Am Heart J. 2012;163(5):844-50 e841.

12. Kalim S, Clish CB, Wenger J, Elmariah S, Yeh RW, Deferio JJ, Pierce K, Deik A, Gerszten RE, Thadhani R, et al. A plasma long-chain acylcarnitine predicts cardiovascular mortality in incident dialysis patients. J Am Heart Assoc. 2013; 2(6):e000542.

13. Rizza S, Copetti M, Rossi C, Cianfarani MA, Zucchelli M, Luzi A, Pecchioli C, Porzio O, Di Cola G, Urbani A, et al. Metabolomics signature improves the prediction of cardiovascular events in elderly subjects. Atherosclerosis. 2014; 232(2):260-4.

14. Stegemann C, Pechlaner R, Willeit P, Langley SR, Mangino M, Mayr U, Menni C, Moayyeri A, Santer P, Rungger G, et al. Lipidomics profiling and risk of cardiovascular disease in the prospective population-based Bruneck study. Circulation. 2014;129(18):1821-31.

15. Alshehry ZH, Mundra PA, Barlow CK, Mellett NA, Wong G, McConville MJ, Simes J, Tonkin AM, Sullivan DR, Barnes EH, et al. Plasma lipidomic profiles improve on traditional risk factors for the prediction of cardiovascular events in type 2 diabetes mellitus. Circulation. 2016;134(21):1637-50.

16. Ganna A, Salihovic S, Sundstrom J, Broeckling CD, Hedman AK, Magnusson PK, Pedersen NL, Larsson A, Siegbahn A, Zilmer M, et al. Large-scale metabolomic profiling identifies novel biomarkers for incident coronary heart disease. PLoS Genet. 2014;10(12):e1004801.

17. Cavus E, Karakas M, Ojeda FM, Kontto J, Veronesi G, Ferrario MM, Linneberg A, Jorgensen T, Meisinger C, Thorand B, et al. Association of circulating metabolites with risk of coronary heart disease in a European population: results from the Biomarkers for Cardiovascular Risk Assessment in Europe (BiomarCaRE) consortium. JAMA Cardiol. 2019;4(12):1270-9.

18. Wang G, Divall S, Radovick S, Paige D, Ning Y, Chen Z, Ji Y, Hong X, Walker SO, Caruso D, et al. Preterm birth and random plasma insulin levels at birth and in early childhood. JAMA. 2014;311(6):587-96.

19. Wang X, Zuckerman B, Pearson C, Kaufman G, Chen C, Wang G, Niu T, Wise $\mathrm{PH}$, Bauchner $\mathrm{H}, \mathrm{Xu}$ X. Maternal cigarette smoking, metabolic gene polymorphism, and infant birth weight. JAMA. 2002;287(2):195-202.

20. ACOG Practice Bulletin No. 202. Gestational hypertension and preeclampsia. Obstet Gynecol 2019, 133(1):e1-e25.

21. Wang TJ, Larson MG, Vasan RS, Cheng S, Rhee EP, McCabe E, Lewis GD, Fox CS, Jacques PF, Fernandez C, et al. Metabolite profiles and the risk of developing diabetes. Nat Med. 2011;17(4):448-53.

22. Rhee EP, Cheng S, Larson MG, Walford GA, Lewis GD, McCabe E, Yang E, Farrell L, Fox CS, O'Donnell CJ, et al. Lipid profiling identifies a triacylglycerol signature of insulin resistance and improves diabetes prediction in humans. J Clin Invest. 2011;121(4):1402-11.

23. Langfelder P, Horvath S. WGCNA: an R package for weighted correlation network analysis. BMC Bioinformatics. 2008:9:559.

24. Rakheja D, Bennett MJ, Foster BM, Domiati-Saad R, Rogers BB. Evidence for fatty acid oxidation in human placenta, and the relationship of fatty acid oxidation enzyme activities with gestational age. Placenta. 2002;23(5):44750.

25. Kenny LC, Broadhurst DI, Dunn W, Brown M, North RA, McCowan L, Roberts C, Cooper GJ, Kell DB, Baker PN, et al. Robust early pregnancy prediction of later preeclampsia using metabolomic biomarkers. Hypertension. 2010;56(4): 741-9.

26. Koster MP, Vreeken RJ, Harms AC, Dane AD, Kuc S, Schielen PC, Hankemeier $T$, Berger R, Visser GH, Pennings JL. First-trimester serum acylcarnitine levels to predict preeclampsia: a metabolomics approach. Dis Markers. 2015;2015: 857108.

27. Thiele IG, Niezen-Koning KE, van Gennip AH, Aarnoudse JG. Increased plasma carnitine concentrations in preeclampsia. Obstet Gynecol. 2004; 103(5 Pt 1):876-80.

28. Erion DM, Shulman Gl. Diacylglycerol-mediated insulin resistance. Nat Med. 2010;16(4):400-2.

29. DeLany JP, Windhauser MM, Champagne CM, Bray GA. Differential oxidation of individual dietary fatty acids in humans. Am J Clin Nutr. 2000;72(4):905-11.

30. Milanski M, Degasperi G, Coope A, Morari J, Denis R, Cintra DE, Tsukumo DM, Anhe G, Amaral ME, Takahashi HK, et al. Saturated fatty acids produce an inflammatory response predominantly through the activation of TLR4 signaling in hypothalamus: implications for the pathogenesis of obesity. J Neurosci. 2009;29(2):359-70.

31. Razquin C, Toledo E, Clish CB, Ruiz-Canela M, Dennis C, Corella D, Papandreou C, Ros E, Estruch R, Guasch-Ferre M, et al. Plasma lipidomic 
profiling and risk of type 2 diabetes in the PREDIMED trial. Diabetes Care. 2018;41(12):2617-24.

32. Ding M, Zeleznik OA, Guasch-Ferre M, Hu J, Lasky-Su J, Lee IM, Jackson RD, Shadyab AH, LaMonte MJ, Clish C, et al. Metabolome-wide association study of the relationship between habitual physical activity and plasma metabolite levels. Am J Epidemiol. 2019;188(11):1932-43.

33. Tabung FK, Balasubramanian R, Liang L, Clinton SK, Cespedes Feliciano EM, Manson JE, Van Horn L, Wactawski-Wende J, Clish CB, Giovannucci EL, et al. Identifying metabolomic profiles of insulinemic dietary patterns. Metabolites. 2019;9(6):120.

34. Razquin C, Liang L, Toledo E, Clish CB, Ruiz-Canela M, Zheng Y, Wang DD, Corella D, Castaner O, Ros E, et al. Plasma lipidome patterns associated with cardiovascular risk in the PREDIMED trial: a case-cohort study. Int J Cardiol. 2018;253:126-32

35. Toledo E, Wang DD, Ruiz-Canela M, Clish CB, Razquin C, Zheng Y, GuaschFerre M, Hruby A, Corella D, Gomez-Gracia E, et al. Plasma lipidomic profiles and cardiovascular events in a randomized intervention trial with the Mediterranean diet. Am J Clin Nutr. 2017;106(4):973-83.

36. Virgiliou C, Gika HG, Witting M, Bletsou AA, Athanasiadis A, Zafrakas M, Thomaidis NS, Raikos N, Makrydimas G, Theodoridis GA. Amniotic fluid and maternal serum metabolic signatures in the second trimester associated with preterm delivery. J Proteome Res. 2017;16(2):898-910.

37. Sharma S, Srivastava VK, Kumar A. Newer N-substituted anthranilic acid derivatives as potent anti-inflammatory agents. Eur J Med Chem. 2002;37(8): 689-97.

38. Duffy JC, Dearden JC, Rostron C. A QSAR study of anti-inflammatory Narylanthranilic acids. J Pharm Pharmacol. 1996;48(9):883-6.

39. Stocker R, Yamamoto Y, McDonagh AF, Glazer AN, Ames BN. Bilirubin is an antioxidant of possible physiological importance. Science. 1987;235(4792): 1043-6.

40. Stocker R, McDonagh AF, Glazer AN, Ames BN. Antioxidant activities of bile pigments: biliverdin and bilirubin. Methods Enzymol. 1990;186:301-9.

41. Abbasi A, Deetman PE, Corpeleijn E, Gansevoort RT, Gans RO, Hillege HL, van der Harst P, Stolk RP, Navis G, Alizadeh BZ, et al. Bilirubin as a potential causal factor in type 2 diabetes risk: a Mendelian randomization study. Diabetes. 2015;64(4):1459-69.

42. Wang L, Bautista LE. Serum bilirubin and the risk of hypertension. Int J Epidemiol. 2015;44(1):142-52.

43. Magnussen EB, Vatten LJ, Lund-Nilsen TI, Salvesen KA, Davey Smith G, Romundstad PR. Prepregnancy cardiovascular risk factors as predictors of pre-eclampsia: population based cohort study. BMJ. 2007;335(7627):978.

44. Harville EW, Viikari JS, Raitakari OT. Preconception cardiovascular risk factors and pregnancy outcome. Epidemiology. 2011;22(5):724-30

45. Egeland GM, Klungsoyr K, Oyen N, Tell GS, Naess O, Skjaerven R. Preconception cardiovascular risk factor differences between gestational hypertension and preeclampsia: cohort Norway study. Hypertension. 2016; 67(6):1173-80

\section{Publisher's Note}

Springer Nature remains neutral with regard to jurisdictional claims in published maps and institutional affiliations.

Ready to submit your research? Choose BMC and benefit from:

- fast, convenient online submission

- thorough peer review by experienced researchers in your field

- rapid publication on acceptance

- support for research data, including large and complex data types

- gold Open Access which fosters wider collaboration and increased citations

- maximum visibility for your research: over $100 \mathrm{M}$ website views per year

At BMC, research is always in progress.

Learn more biomedcentral.com/submissions 